\title{
Gut sterilization in experimental colitis leukocyte mediated colon injury, and effects on angiogenesis/lymphangiogenesis"
}

\author{
Mihir Patel ${ }^{1}$, Justin Olinde ${ }^{1}$, Allison Tatum ${ }^{1}$, Chaitanya V. Ganta ${ }^{1}$, Walter E. Cromer ${ }^{2}$, \\ Kenneth Manas ${ }^{3}$, Christopher P. Monceaux ${ }^{1}$, J. Steven Alexander ${ }^{1}$ \\ ${ }^{1}$ Department of Molecular and Cellular Physiology, LSU Health Shreveport, Shreveport, USA \\ ${ }^{2}$ Department of Cellular Biology and Anatomy, LSU Health Shreveport, Shreveport, USA \\ ${ }^{3}$ Department of Gastroenterology and Hepatology, LSU Health Shreveport, Shreveport, USA \\ ${ }^{4}$ Department of Microbiology and Immunology, LSU Health Shreveport, Shreveport, USA \\ Email: jalexa@1suhsc.edu
} Ankur R. Sheth ${ }^{3}$, Merilyn H. Jennings ${ }^{1}$, J. Michael Mathis ${ }^{2}$, Traci Testerman ${ }^{4}$, Paul A. Jordan ${ }^{3}$,

Received 6 December 2012; revised 4 January 2013; accepted 11 January 2013

\section{ABSTRACT}

Inappropriate responses to normal commensal bacteria trigger immune activation in both inflammatory bowel disease and experimental colitis. How gut flora contribute to the pathogenesis of inflammatory bowel disease is unclear, but may involve entrapment of leukocytes and remodeling of the vascular system. Here we evaluated how the progression and tissue remodeling in experimental colitis differ in a germfree model of mouse colitis. Four treatment groups were used: control, antibiotic-treated (ABX), dextran sulfate colitis (DSS) and DSS pre- and co-treated with antibiotics (DSS $+\mathrm{ABX}$ ). In days 0-3 of the study, germ-free mice received antibiotics (vancomycin, neomycin, and metronidazole). During the next 11 days, antibiotics were continued and DSS (3\%) added to "colitis" groups. Disease activity, weight, stool form and blood were monitored daily. Mice were sacrificed and tissue samples harvested. Histopathological scores in controls (0.00) and in $\mathrm{ABX}(\mathbf{1 . 0}+/-\mathbf{0 . 8 1})$ were significantly $(p<0.001)$ lower than DSS $(12+/-0)$. Extents of injury, inflammation and crypt damage were all improved in DSS + ABX. The Disease Activity Index score (day 11) was significantly worse in the DSS group compared to the DSS + ABX group. Stool blood and form scores were also significantly improved among these groups. Importantly, myeloperoxidase was significantly reduced in DSS + ABX, indicating that neutrophil infiltration was blocked. Colitis was associated with an increase in blood and lymphatic vessels; both of these events were also significantly reduced by gut sterilization. Our experiment shows that clinical and histopathological severity of colitis was significantly worse in the DSS colitis

*This work was funded by PR100451, NIH grant HL47615-17 and the Feist Cardiovascular Research Trust. group compared to the DSS + ABX group, supporting the hypothesis that development of IBD is likely to be less severe with appropriate antibiotic treatment. In particular, gut sterilization effectively reduces leukocyte-dependent (PMN) injury to improve outcomes and may be an important target for therapy.

Keywords: Crohn's Disease; Antibiotics;

Myeloperoxidase; Lymphatics; Angiogenesis

\section{INTRODUCTION}

Inflammatory Bowel Diseases (IBD) are a group of gastrointestinal diseases including Crohn's disease (CD) and ulcerative colitis (UC). CD is characterized by granulomas, skipped lesions, transmural inflammation, goblet cell hyperplasia, fistulae and luminal stenosis involving any part of the GI tract. UC is characterized by mucosal inflammation, pseudopolyps, reduced goblet cells, absence of fistulization, stenosis, and granulomas, and is restricted to the colon. Several factors which can increase the risk for IBD include genetic background, stress, diet, and infections (e.g. atypical Mycobacteria) [1]. Recent increases of IBD incidence in developing nations and nonclassical populations [2] also suggest socioeconomic status and environmental factors (e.g. dietary "hygiene", and "cold chain") may contribute to IBD etiology [1].

The etiology of CD and UC appears to represent inappropriate immune responses to normal gut bacteria which occur in genetically susceptible individuals. IBD associated genetic polymorphisms like the Nod2/Card15 locus [3] hyperactivate immune system responsiveness and reduce tolerance to intestinal flora, with sustained gut inflammation, fibrotic changes, and destruction of the intestinal mucosal barrier. Based on these findings, one potential IBD therapy could be reduction of the enteric 
flora. This could reduce immune responses and be less toxic and more focused than immunosuppressive drugs [4].

Gut flora contributes to the etiopathology of animal models of IBD as well as human CD and UC. Several experimental immune models (HLA-B27, Samp1/Yit, TGF-bRII and IL-10-/-, TNFDARE) and erosive models (iodoacetamide, picrylsulfonic acid, TNBS, dextran sulfate sodium-DSS) have been used to study IBD mechanisms in animals. Both human and animal models of IBD suggest that barrier dysfunction is evident before disease onset, and contributes to development of these diseases [5]. DSS, a negatively charged dextran, disturbs gut barrier, allowing bacteria to penetrate the gut lamina propria, and is used as an experimental model of colitis. Similarly, iodoacetamide and TNBS also disturb gut barrier leading to inflammation [6]. Additionally, IBD genetic models [7] exhibit dysregulated gut barrier, which precedes the onset of IBD activity, and importantly display reduced inflammation following antibiotic therapy. Similar prodromal changes in gut epithelial barrier are also seen in human IBD [8,9]. Results in animal experimental models and in human trials using broad spectrum antibiotics (e.g. metronidazole plus ciprofloxacin) have demonstrated therapeutic success [10]. Thus, despite disturbances in gut barrier, reduced enteric bacterial loads reduce or eliminate inflammation and play an important role in IBD and therapy.

However, commensal enteric bacteria also have a wide range of beneficial effects in the GI tract, including digestion of complex carbohydrates, control of the intestinal mucosal barrier, modulation of inflammation, and regulating the activity of the enteric nervous system [11]. The gut vascular angiogenesis, in particular, is also known for development of gut injury in IBD, but whether and how gut commensals contribute to vascular disturbances in IBD is not known. Further, while angiogenesis is enhanced in IBD, whether or how the immune system and intestinal flora may modulate lymphangiogenesis is unclear [12].

Here we studied how complete gut sterilization alters disease activity, weight changes, histopathology of experimental IBD, and various inflammatory parameters like leukocyte infiltration, angiogenesis, and lymphangiogenesis. We analyzed these disease features in four different groups of mice: control, antibiotic treated (ABX), dextran sulfate colitis (DSS), and DSS pre and co treated with antibiotics (DSS $+\mathrm{ABX}$ ). Importantly, as previous studies have linked IBD with changes in angiogenesis and lymphangiogenesis, our study demonstrates how commensal bacteria and broad spectrum antibiotics affect both angiogenesis and lymphangiogenesis in the 3\% DSS model, and further shows the protective role of lymphatic expansion.

\section{MATERIALS AND METHODS}

\subsection{Experimental Groups}

Mice used in this study were male or female KBLACZ mice. The mice used in these experiments were 5 - 9 weeks of age, and had an average initial weight of 20.84 $+/-1.1(\mathrm{SE})$ gms on day 0 . A total of 4 groups were selected: control $(\mathrm{n}=4), \operatorname{ABX}(\mathrm{n}=4)$; DSS $(\mathrm{n}=5)$; and $\mathrm{DSS}+\operatorname{ABX}(\mathrm{n}=5)$. Mice in different groups were separated into different cages. All mice were fed autoclaved alfalfa mouse chow and sterile drinking water and data was recorded of consumption each day. Animal protocols were reviewed and approved by the LSUHSC and University of Arizona Institutional Animal Care and Use Committees (IACUC).

\subsection{Induction of DSS Colitis and Antibiotic Treatment}

The experiment was divided in 2 phases: In the first phase from day -3 to 0 , mice in the ABX and DSS + ABX group received antibiotics preemptively in their drinking water to sterilize the gut. The control and DSS groups received water only. The antibiotic solution used in the first 3 days consisted of $500 \mathrm{mg} / \mathrm{L}$ vancomycin, $350 \mathrm{mg} / \mathrm{L}$ neomycin and $600 \mathrm{mg} / \mathrm{L}$ metronidazole (to eliminate Enterococci, gram-negatives, and anaerobes (Bacteroides/Clostridium respectively) [13,14]. In the second phase (Day 0 to 11): On the 0 day, the antibiotic concentration in water was reduced by $50 \%$, and $3 \%$ DSS [as described, Soriano et al.], ad libitum (DSS, MW 1/4 36 - $50 \mathrm{kDa}$; ICN Biomedicals, Costa Mesa, CA) was added to the drinking water of the DSS and DSS + ABX group for the duration of the study [9]. At 3 - 4 days, progressive weight loss, diarrhea, occult blood, leukocyte infiltration, colon shortening, loss of intestinal epithelial barrier, and histopathological changes in colon structure were observed. Mouse weight, stool form, occult blood, food (grams), and liquid $(\mathrm{mL})$ consumption were recorded daily.

\subsection{Drug Consumption}

Table 1 shows the drug consumption in ABX and DSS + ABX group over 14 days. The first phase includes from day -3 to day 0 , and second phase includes from day 1 to day 11. During the first phase, antibiotics were given with drinking water, and in the second phase antibiotic concentration was halved in the DSS + ABX group after initiation of $3 \%$ DSS administration. The antibiotic concentration used in this experiment was $500 \mathrm{mg} / \mathrm{L}$ of vancomycin, $350 \mathrm{mg} / \mathrm{L}$ of neomycin and $600 \mathrm{mg} / \mathrm{L}$ of metronidazole. The main issue with the combination of antibiotics is the toxicity of the regimen, but the concentration of 
Table 1. Antibiotic loading from days -3 to day 0 and days 1 to 11. First value is $\mathrm{mg} / \mathrm{kg} /$ day in pretreatment and second value is $\mathrm{mg} / \mathrm{kg} /$ day during colitis induction phase. Values are based on medicated water consumption per day/cage of mice.

\begin{tabular}{cccc}
\hline \multirow{2}{*}{ Treatment groups } & \multicolumn{3}{c}{ Antibiotics consumption } \\
& (doses in $\mathrm{mg} / \mathrm{kg} /$ day) (day -3 to 0/day 1 to 11) \\
\cline { 2 - 4 } & Neomycin & Vancomycin & Metronidazole \\
\hline $\mathrm{ABX}(\mathrm{n}=4)$ & $29.75 / 27.91$ & $42.50 / 39.88$ & $51.00 / 47.85$ \\
$\mathrm{DSS}+\mathrm{ABX}(\mathrm{n}=5)$ & $31.02 / 23.82$ & $44.32 / 34.02$ & $53.18 / 40.84$ \\
\hline
\end{tabular}

antibiotics was lower than LD 50. The dosages are calculated with average daily consumption of antibiotics per average initial weight of mice.

\subsection{Necropsy}

At the end of the study, mice were anesthetized with a Ketamine $(50 \mathrm{mg} / \mathrm{ml})$ and Xylazine $(2.85 \mathrm{mg} / \mathrm{ml})$ mixture; mice were sacrificed and blood and tissue specimens were collected. The ceca were removed and colon length and weight were measured. Spleen and cecum weights were also recorded. Mice were then sacrificed by cardiac puncture (under ketamine/xylazine anesthesia). Histological samples were fixed in cold $3.7 \%$ phosphatebuffered formalin, or frozen at $20 \mathrm{C}$ for myeloperoxidase (MPO) and Western blotting analysis.

\subsection{Gut Sterilization}

To determine that this level of combined antibiotics was sufficient to eliminate gut flora, fecal samples $(100 \mu \mathrm{l}$ of 1 gms mouse feces dispersed in $1 \mathrm{ml}$ water) were plated onto $1.5 \%$ selective agar plates. These plates contained the combined antibiotic regimen at concentrations equivalent to the levels of antibiotics consumed by the mice or nutrient agar without antibiotics. Plates were incubated at $37^{\circ} \mathrm{C}$ overnight and resulting colonies were counted.

\subsection{Evaluation of Clinical Colitis}

Body weight, stool form, and occult blood were scored daily as described [15] and disease activity index (DAI) was determined as the average of these scores: 1) weight change (calculated as: percent difference between originnal body weight and weight on any given day with a score between 0 and 4) (0:<1\%, 1: $1 \%-5 \%, 2: 5 \%$ $10 \%, 3: 10 \%-15 \%, 4:>15 \%) ; 2)$ stool consistency score based on qualitative examination ( 0 - very firm, dry, non-adherent; 1 -firm, moist, adherent; 2-soft, very adherent; 3-very soft, pliable; 4-formless, liquid); 3) occult blood score based on results using "Colo-screen" kits (Helena Labs, Beaumont, TX): (0-no color development, 1-greenish blue reaction, 2-consistent blue color, 3-rust color stools + blue reaction, 4-wet blood + dark blue reaction) [16-19].

\subsection{Histopathological Analysis}

Formalin-fixed colon sections were paraffin embedded and $10 \mu \mathrm{m}$ sections stained with hematoxylin/eosin. Slides were analyzed for evidence of histopathological injury using criteria established by Cooper et al. [20]. This system includes edema, extent of injury, leukocyte infiltration, crypt abscesses and loss of goblet cells. These were scored on inflammation severity ( 0 - none, 1-slight, 2-moderate, 3-severe), extent of injury (0-none, 1-mucosal, 2-mucosal + submucosal, 3transmural), and crypt damage ( 0 -none, 1 -basal $1 / 3$ damaged, 2-basal 2/3 damaged, 3-only surface epithelium intact, 4-loss of entire crypt and epithelium). Each value was multiplied by an extent index which reflects the amount of involvement for each section (x1: $0 \%-25 \%, x 2: 26 \%-50 \%, x 3: 51 \%-75 \%$, x $4: 76 \%-$ $100 \%)$. The final score, based on at least three different colon samples, were analyzed and equaled the sum of the individual extent-adjusted scores [20]. A maximum possible histopathological score for this assay is 40 .

\subsection{Measurement of Tissue MPO Content}

MPO activity was measured as described by Grisham et al. [21], and as modified by Hausmann et al. [22]. $25 \mathrm{mg}$ samples of colon tissue were frozen under N2 [23], crushed and freeze-thawed $3 \mathrm{X}$ in $0.5 \%$ HETAB buffer, sonicated for 10 seconds at $50 \%$ max power [24], and cleared by centrifugation $(10,000 \times \mathrm{g}, 5 \mathrm{~min})$ before MPO activity of supernatants was measured (using $0.1 \%$ o-dianisidine substrate) at an absorbance of $450 \mathrm{~nm} / \mathrm{min} / \mathrm{mg}$ tissue.

\subsection{Immunohistochemistry}

Colon sections fixed $>12 \mathrm{~h}$ in $3.7 \%$ phosphate buffered formalin were embedded in paraffin. Sections $(10 \mu \mathrm{m})$ were collected onto Superfrost-Excell slides, and deparaffinized prior to antigen retrieval. Slides were incubated in primary antibody $(1: 125,1 \mathrm{hr})$, washed in $0.1 \%$ Bovine serum albumin (BSA), reacted in HRP-conjugated secondary antibody $(1: 2500,1 \mathrm{~h})$, and reacted with DAB/peroxide (5 mins). The $3.7 \%$ phosphate-buffered formaldehyde fixed tissue sections $(5 \mu \mathrm{m})$ were immunostained using $\mathrm{DAB} /$ peroxidase for vascular endothelial growth factor receptor-3 (VEGFR-3) (lymphatic vessels) and MECA-32 (mouse endothelial cell antigen-32) monoclonal antibody [25,26], a blood vascular specific marker [26] obtained from Developmental Hybridoma Studies Bank (Iowa City, IA) and prepared on-site. Slides were hematoxylin counterstained (30 s) and sealed in Permount.

\subsection{Trans Endothelial Electrical Resistance (TEER)}

24 well PETP (polyethylene terephthalate) transwell in- 
serts (BD Biosciences; San Jose, CA) were coated with $2 \%$ gelatin and seeded with young adult mouse colon cells (YAMCs) in RPMI medium with 5\% FCS, $1 \%$ insulin/transferrin/selenite and $5 \mathrm{U} / \mathrm{ml} \mathrm{INF}-\gamma$ and allowed to reach confluence. At confluence, medium was removed and replaced with experimental RPMI media containing $3 \%, 1 \%$ or $0.5 \%$ DSS or control medium in both upper and lower chambers. Resistance readings were taken at $0,1,2,18$ and 24 hours using an epithelial volt-ohmmeter (World Precision Instruments; Sarasota, FL) and data were converted to percent initial resistance. Data were analyzed by repeated measures ANOVA with Dunnett's post-hoc test to determine significant $(\mathrm{p}<0.05)$ departure from basal resistance.

\subsection{Effect of DSS on Colonic Epithelial Metabolism}

YAMCs were plated in 96 well plates coated with $2 \%$ porcine gelatin in RPMI and allowed to grow to confluence. When cells reached confluence, the culture medium was removed and replaced with experimental RPMI media containing $3 \%, 1 \%$ or $0.5 \%$ DSS or control medium. To determine the effect of DSS on metabolism, each of the groups was divided in two $(n=10)$ and either treated for eight hours with DSS or treated for eight hours with DSS followed by a 24 hour recovery period in RPMI culture medium. Upon the end of treatment, media was changed to RPMI without phenol red containing $0.5 \mathrm{mg} / \mathrm{mL}$

(3-(4,5-Dimethylthiazol-2-yl)-2,5-diphenyltetrazolium bromide) (MTT) for two hours. Next, medium was aspirated and converted MTT was released by addition of acidified isopropanol. Absorbance was measured at 570 $\mathrm{nm}$ with background subtraction at $650 \mathrm{~nm}$. Results were analyzed by one-way ANOVA with Dunnett's post-hoc test.

\subsection{Statistical Analysis}

Disease activity studies were evaluated using repeatedmeasures ANOVA with Dunnett's post testing, Immunohistochemistry, macroscopic, and microscopic data were analyzed with one way ANOVA with Tukey Kramer multiple comparison test. MPO data were analyzed by 1 way ANOVA with Dunnett's post testing (Graph Pad In stat 3 software, San Diego, CA).

\subsection{Microphotography}

Images of immunostained tissue sections were photographed using Nikon bright field microscopy station (Olympus CK2) and analyzed by a double-blinded expert for the numbers of blood and lymphatic vessels and vessel dimensions using Image-J (NIH, Bethesda, MD).

\section{RESULTS}

\subsection{Gut Sterilization}

We found that the antibiotic treatment used in this study was sufficient to prevent the growth of any colonies on nutrient agar. This appeared to be both bactericidal and bacteriostatic since feces from mice undergoing antibiotic water treatment were found to contain sufficient bacteria to yield viable 24 nutrient broth cultures (data not shown).

\subsection{Disease Activity}

Antibiotics reduce the disease activity: Disease activity represents the development of cumulative symptoms (weight change, stool consistency, and occult blood). The DSS group showed onset of disease activity on day 3 ( $p$ $<0.01^{* *}$ ) and it continually increased toward day 11 , while the antibiotic-treated DSS group (DSS + ABX) showed a similar onset initially, but after day 5 , the disease activity substantially slowed (Figure 1(a)). The ABX group showed mild disturbances, but did not develop colitis.

Weight of the mice: Body weight remained constant in the control group and increased gradually in the ABX group $\left(\mathrm{p}<0.01^{* *}\right)$. The DSS group was characterized by a consistent weight loss $\left(\mathrm{p}<0.01^{* *}\right)$, as expected in the colitis model (Figure 1(b)). Conversely, the weight of the mice in the DSS + ABX group remained fairly constant compared to the control group ( $\mathrm{p}>0.05)$.

Stool consistency: (Figure 1(c)) In the DSS group, diarrhea started on Day 1, immediately after the DSS administration and it worsened throughout the course of the study, whereas the DSS + ABX group demonstrated initial diarrheal symptoms on Day 2, but remained less severe than the DSS group ( $p<0.01)$. The ABX and control group had no severe diarrheal symptoms.

\subsection{Macroscopic Findings}

Antibiotics prevents inflammatory shortening of colon \& weight change: Due to severe inflammation in DSStreated mice (Figure 2(a)), the colon length was much shorter $(7.0+/-0.7 \mathrm{~cm})$ than that of the control group $(5.5+/-0.52 \mathrm{~cm})$ at $\mathrm{p}<0.01$. Antibiotics preserved the colon length of DSS + ABX group $(6.2+/-0.5 \mathrm{~cm} ; \mathrm{p}>$ 0.05). Inflammation induced swelling of the colon (Figure 2(b)), which caused an increase in the DSS mice colon weight $(0.27+/-0.05 \mathrm{~g})$ compared to the control $(0.19+/-0.03 \mathrm{~g})$ at $\mathrm{p}<0.01$. Whereas, antibiotics prevented the swelling of the DSS $+\mathrm{ABX}$ group colons $(\mathrm{p}>$ 0.05).

Spleen weights: The spleen weight may be an indirect index of immune response induced by inflammation. The DSS mice group exhibited an increase in spleen weight 


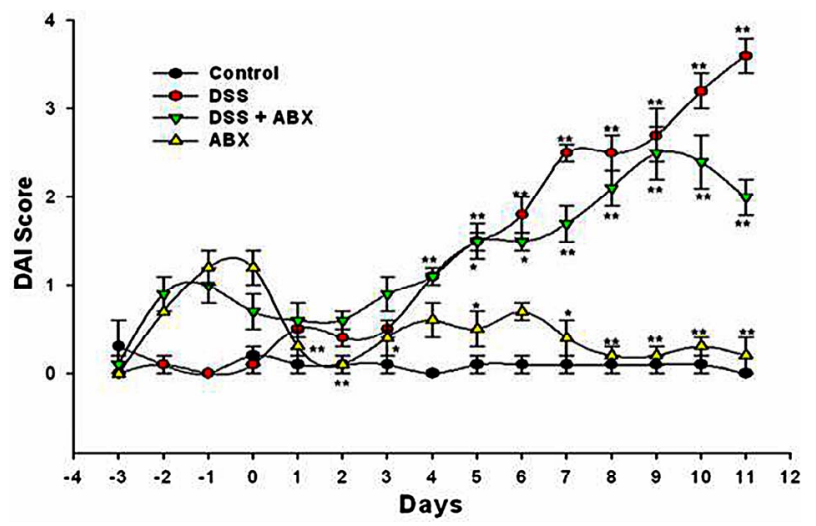

(a)

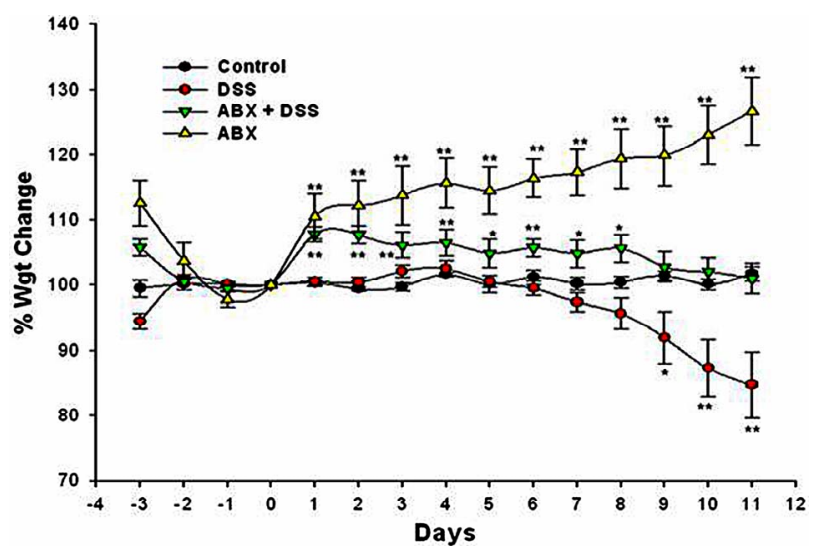

(b)

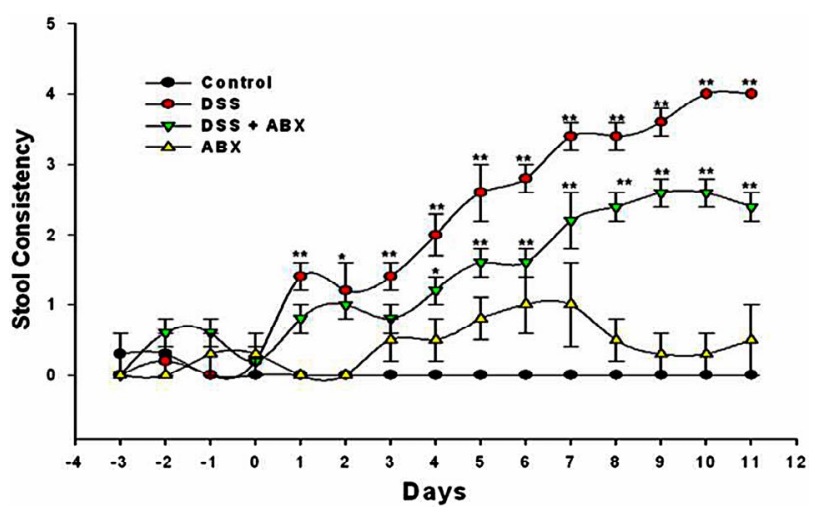

(c)

Figure 1. (a) Disease activity index was calculated with a cumulative score of weight change, stool form, and occult blood. The DSS group displayed significant elevation of DAI from baseline $(\mathrm{p}<0.01)$ at Day 5 throughout Day 11; though DSS + ABX DAI scores differed from baseline, they were substantially lower than DSS; (b) Over 14 days, weight loss in DSS mice was maximum compared to the control $(\mathrm{p}<0.01)$, while DSS + ABX group and control group had similar weight changes at Day 11; (c) Stool consistency (diarrhea) was significantly improved in the DSS + ABX group compared to DSS over 11 days $(\mathrm{p}<0.01)$.

$(0.14+/-0.005 \mathrm{~g})$ compared to control $(0.05+/-0.005 \mathrm{~g}$; $\mathrm{p}<0.01)$, and although the DSS + ABX group also ap- peared to be increased, this change was not statistically significant $(0.10+/-0.03 \mathrm{~g} ; \mathrm{p}>0.05)$. This justifies the ablation in inflammatory immune response. The weights of the ABX group were similar to the control $(0.045+/-$ $0.002 \mathrm{~g} ; \mathrm{p}>0.05$ ). This eliminates the possibility of antibiotics playing a role in spleen weight change in control conditions (Figure 2(c)).

Colon cross-sectional area: The cross-sectional area indicates the morphological changes induced by inflamemation to destroy the integrity of colon. The DSS group demonstrated mean cross-sectional area (Figure 2(d)) of $30.8+/-6.7 \mathrm{~mm}^{2}$, which was higher than any of the other groups at $\mathrm{p}<0.001$. Antibiotics preserved crosssectional areas of the ABX/DSS group (20.1 +/- 5.98 $\mathrm{mm}^{2}$ ) because this group did not differ from the control or ABX groups (mean cross-sectional area of $21.03+/-$ 4.83 and $18.20+/-3.77$, respectively; $p>0.05$ ).

\subsection{Histopathological Colonic Injuury}

Histopathological analysis was based on H\&E stained colon sections under light microscopy scored by an expert with double blind experimental conditions. The total histopathology score (Figure 3(a)) includes 3 parameters: severity of inflammation (Figure 3(b)), extent of injury (Figure 3(c)), and crypt damage (Figure 3(d)). The control group demonstrated no notable epithelial lesions on light microscopy (mean total score $0+/-0$ ), whereas the DSS group displayed substantial histological damage (38 $+/-1.581, \mathrm{p}<0.001$ ), including focal erosions of the epithelium, crypt dilation, and acute inflammatory infiltrates of lymphocytes and granulocytes in the sub-epithelium and lamina propria (Figure 3(e)). Vascular congestion and goblet cell loss, in conjunction with moderate to severe edema, was also observed in this group. All 3 parameters of histopathological score increased significantly in DSS at $\mathrm{p}<0.001$, compared to controls. Meanwhile, antibiotics were able to reduce inflammatory colon damage in the DSS + ABX group $(15.2+/-3.27$; $\mathrm{p}$ $<0.001)$. In all 3 parameters, however, it did differ considerably from the control group $(\mathrm{p}<0.001)$.

\subsection{Colonic Angiogenesis}

Blood vascular density (Figure 4(a)) indicates the neovascularization secondary to acute inflammation. The DSS group showed growth of new vessel formation with a mean count of $84.37+/-7.20$ vessels per section, which was the only group with any change from control at $p<$ 0.001 . Antibiotics suppressed vessel counts 2.02 times lower for the ABX/DSS group (41.66+/- 5.34 vessels per section) and it is approximately identical to the control group $(40.90+/-10.93$ vessels per section; $p>0.05)$ and the ABX group (mean $30.16+/-7.81 ; \mathrm{p}>0.05$ ). 


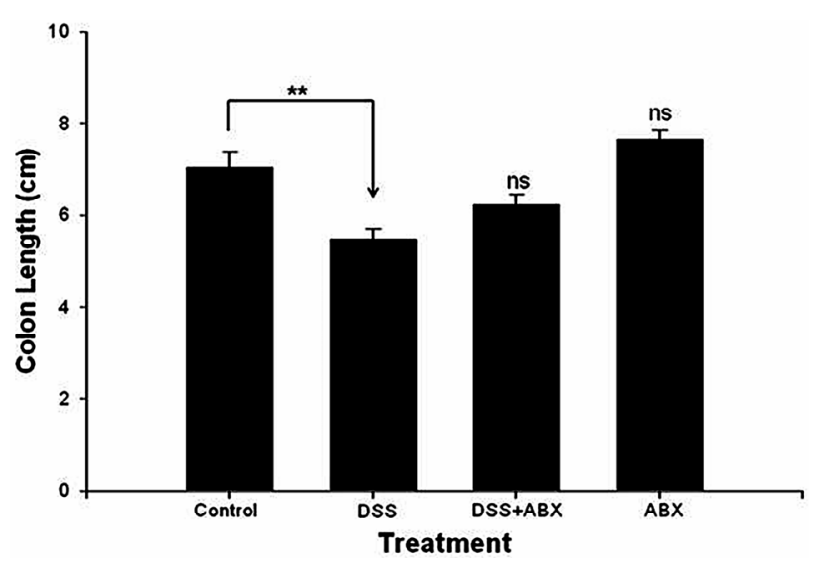

(a)

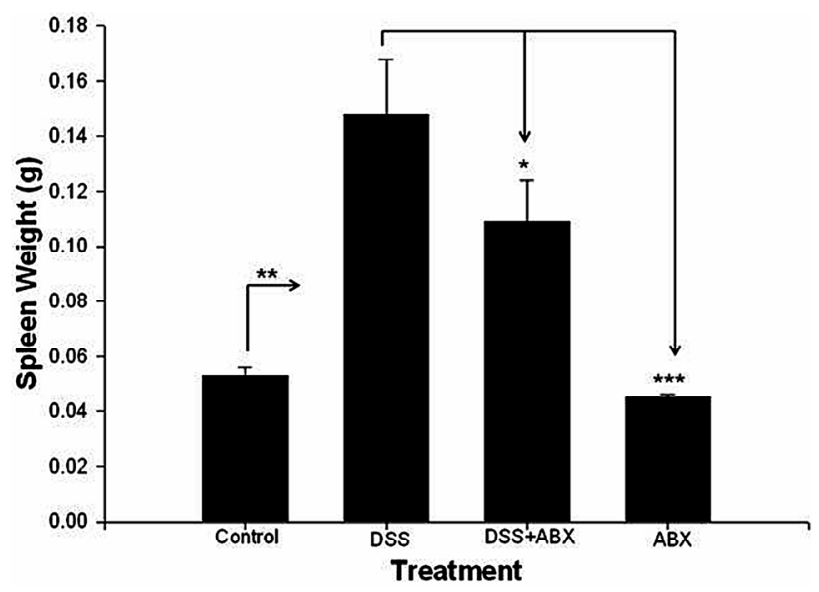

(c)

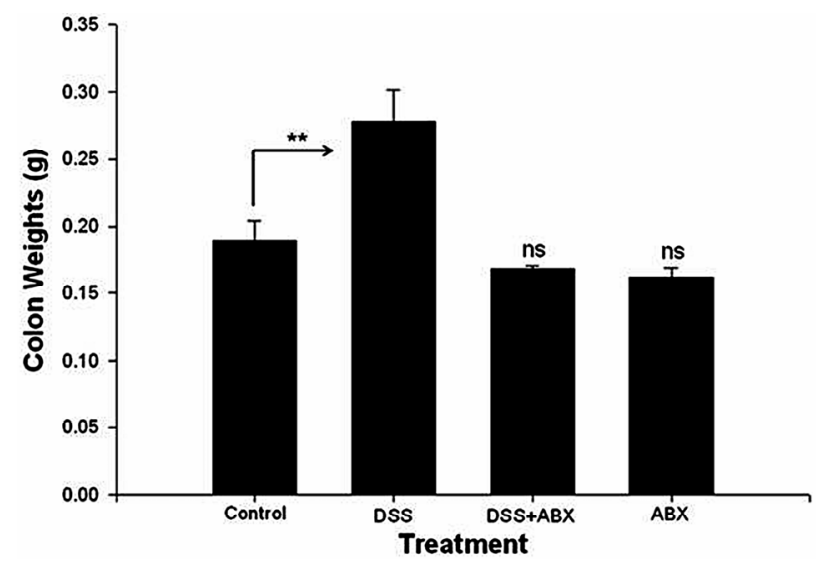

(b)

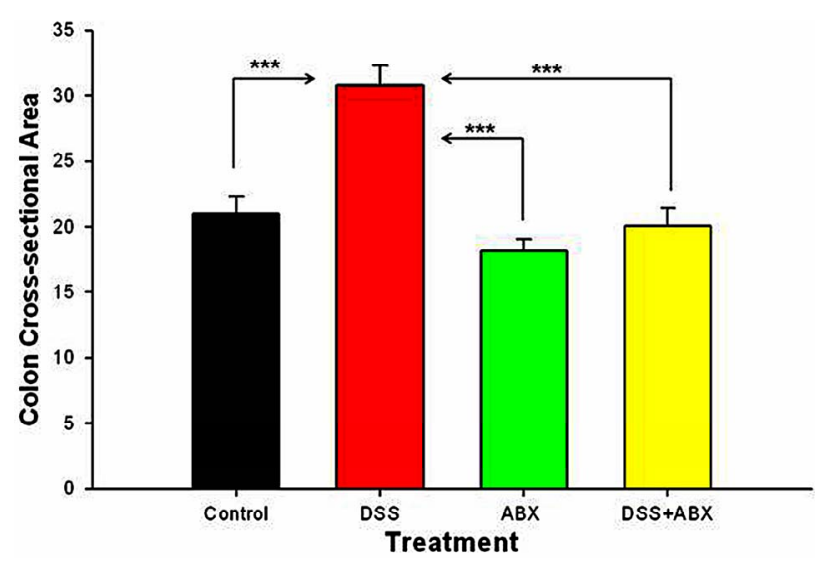

(d)

Figure 2. (a) Antibiotics prevent colon shortening: DSS induced inflammatory colon shortening was exacerbated in DSS (5.48 $+/-$ $0.52 \mathrm{~cm})$ compared to controls $(7+/-0.7 \mathrm{~cm} ; \mathrm{p}<0.01)$. Antibiotics preserved the colon length in DSS $+\mathrm{ABX}(6.22+/-0.5 \mathrm{~cm}) ;(\mathrm{b})$ Colon weight: Edematous swelling of the colon caused the weight gain in DSS $(0.27+/-0.05 \mathrm{gm})$; this significantly differs from control $(0.18+/-0.02 \mathrm{gm} ; \mathrm{p}<0.01)$, while DSS + ABX $(0.16 \mathrm{gm})$ was identical to control; (c) Effects of antibiotics on spleen weight: DSS + ABX $(0.108+/-0.03$ gm) and DSS $(0.14+/-0.04)$ showed splenic enlargement, which is elevated from baseline controls $(0.005+/-0.005 \mathrm{gm})$; (d) Colon cross-sectional area: DSS with colonic inflammation had higher cross-sectional area than control (p $<0.001)$. Antibiotics prevent disruption of the integrity of colon architecture in DSS + ABX $\left(20.1+/-5.98 \mathrm{~mm}^{2}\right)$, which did not differ from the control or $\mathrm{ABX}$ (mean cross-sectional area of $21.03+/-4.83$ and $18.20+/-3.77$, respectively; $\mathrm{p}>0.05$ ).

\subsection{Colonic Lymphangiogenesis}

Lymphatic density (Figure 4(b) indicates immune reactivity to inflammation by neo-lymphangiogenesis. The DSS group showed prominent lymphatic vessel proliferation in the mucosa, lamina propria, and sub-mucosa of the colon (113.25+/- $14.05 \mathrm{vps})$. Conversely, with the effect of protective antibiotics, the ABX/DSS group is 1.74 times lower than the DSS group $(64.86+/-4.95, \mathrm{p}<$ $0.001)$. The control group $(24.45+/-6.85)$ and the antibiotic group $(32.38+/-10.28)$ were essentially identical.

\subsection{Comparison of Colonic Angiogenesis Lymphaniogenesis}

Blood vascular density and lymphatic proliferation are parallel parameters of inflammation. This experiment shows how antibiotics suppress the native immune response by hindering the development of angiogenesis and lymphangiogenesis (Figure 4(c)). The DSS colitis group showed the normal immune response to chemical (DSS) induced inflammation by having the highest number on vessel counts compared to the control group at $p<$ 0.001 . Interestingly, angiogenesis in the ABX + DSS is identical to the controls, while lymphangiogenesis is 2.1 fold higher than baseline. This indicates that the antibiotics play different roles in suppression of inflammatory angiogenesis \& lymphangiogenesis.

\subsection{MPO Activity}

Myeloperoxidase is a peroxidase enzyme present in neutrophil granulocytes, which indicates neutrophilia during 


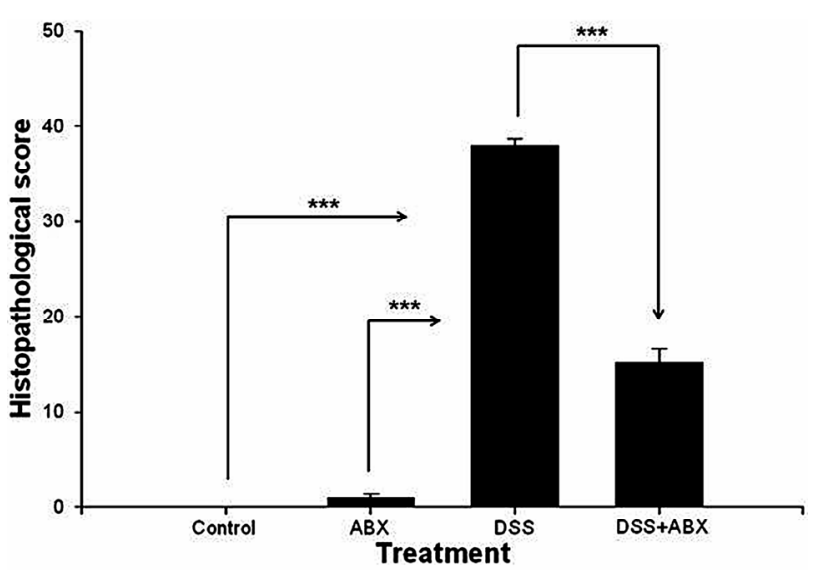

(a)

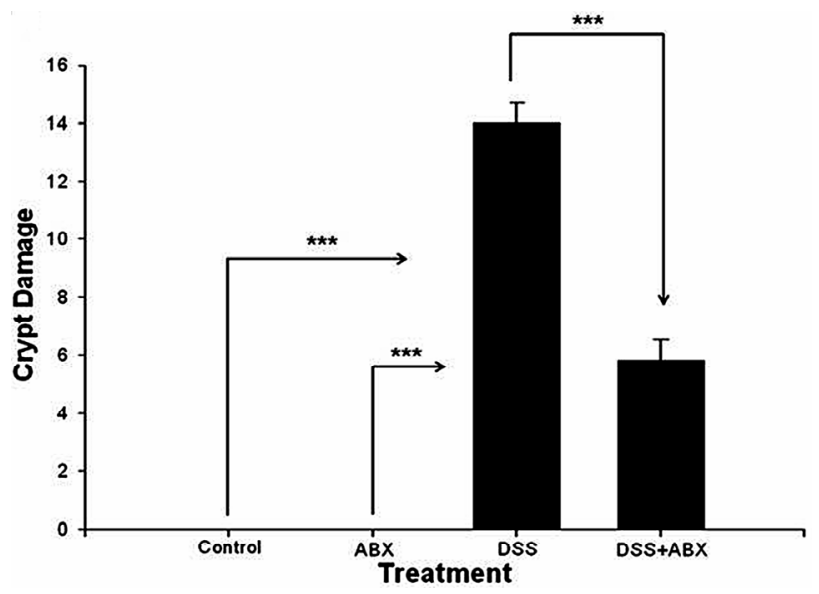

(c)
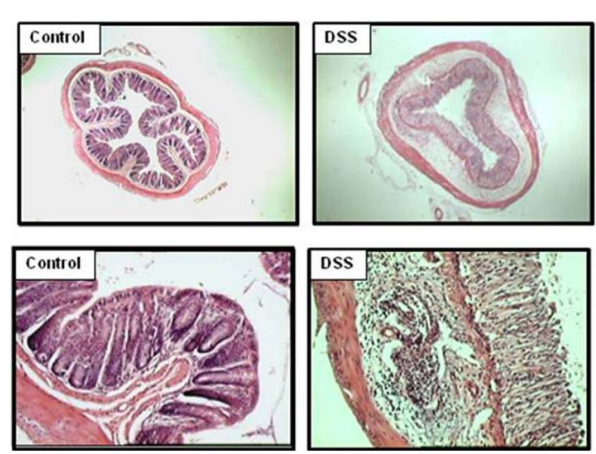

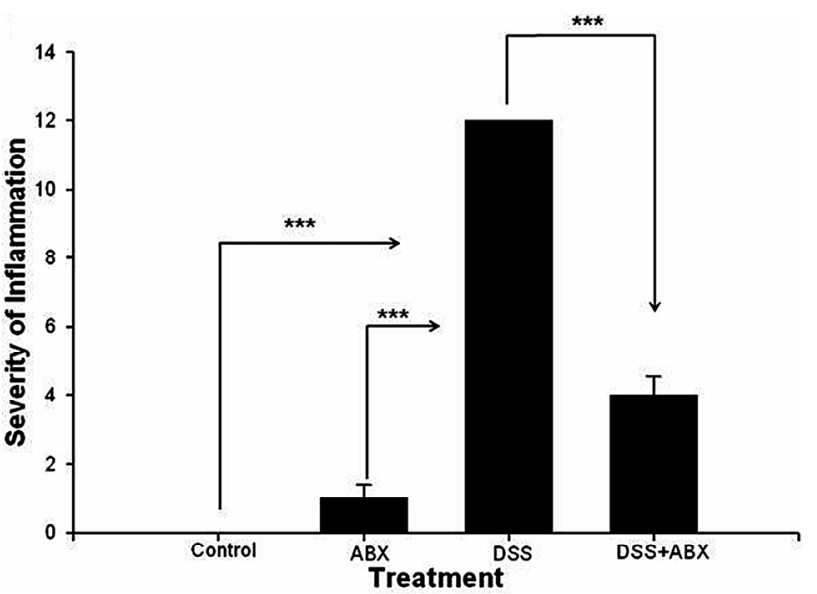

(b)

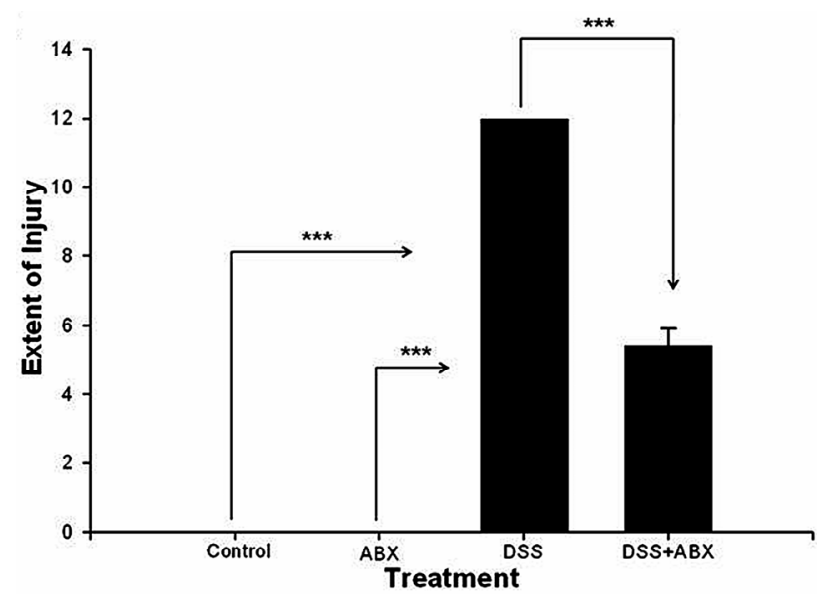

(d)
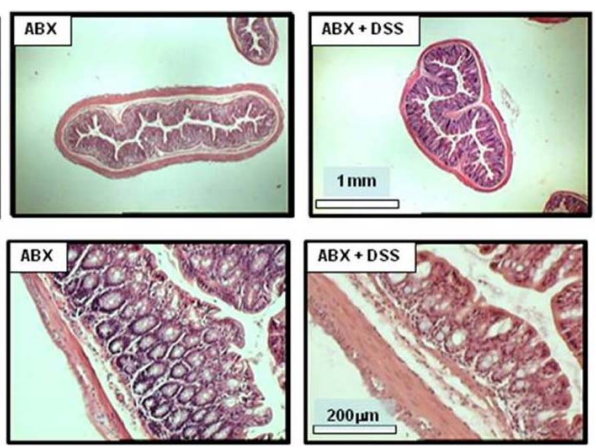

(e)

Figure 3. (a) Histopathological Scores: The DSS group had a cumulative histopathological score of $38+/-1.5$ (normal score $=0$, max injury $=40$ ), which was significantly elevated compared to the controls. The DSS + ABX group was also elevated but less so than the DSS group; (b) Histopathological Scores: The DSS group had a significantly higher severity of inflammation score than the control, ABX, and DSS + ABX groups; (c) Histopathological Scores: The DSS group had a significantly higher crypt damage score than the control, ABX, or DSS + ABX groups; (d) Histopathological Scores: The DSS group had a significantly higher extent of injury score than the control, ABX, or DSS + ABX groups; (e) Pictures of various slide photographs of H\&E staining of control, DSS, $\mathrm{ABX}$ and DSS + ABX groups.

acute inflammation. The DSS group exhibited the highest amount of neutrophil infiltration, as measured by myeloperoxidase (MPO) activity (Figure 5), with a mean absorbance change of $0.19+/-0.15$ and a significant de- viation from control $(0.02+/-0.006 ; \mathrm{p}<0.05)$. The ABX/ DSS group $(0.045+/-0.02)$ and the ABX group $(0.01$ $+/-0.006)$ didn't produce any significant change from control $(\mathrm{p}>0.05)$. 

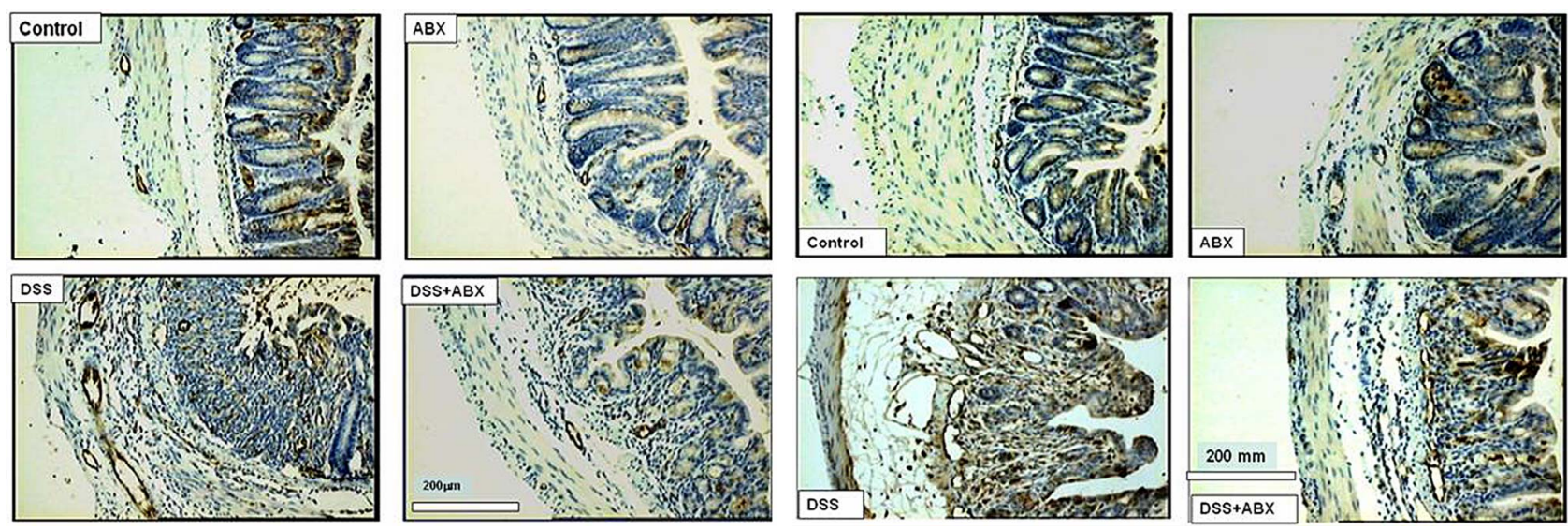

(a)

(b)

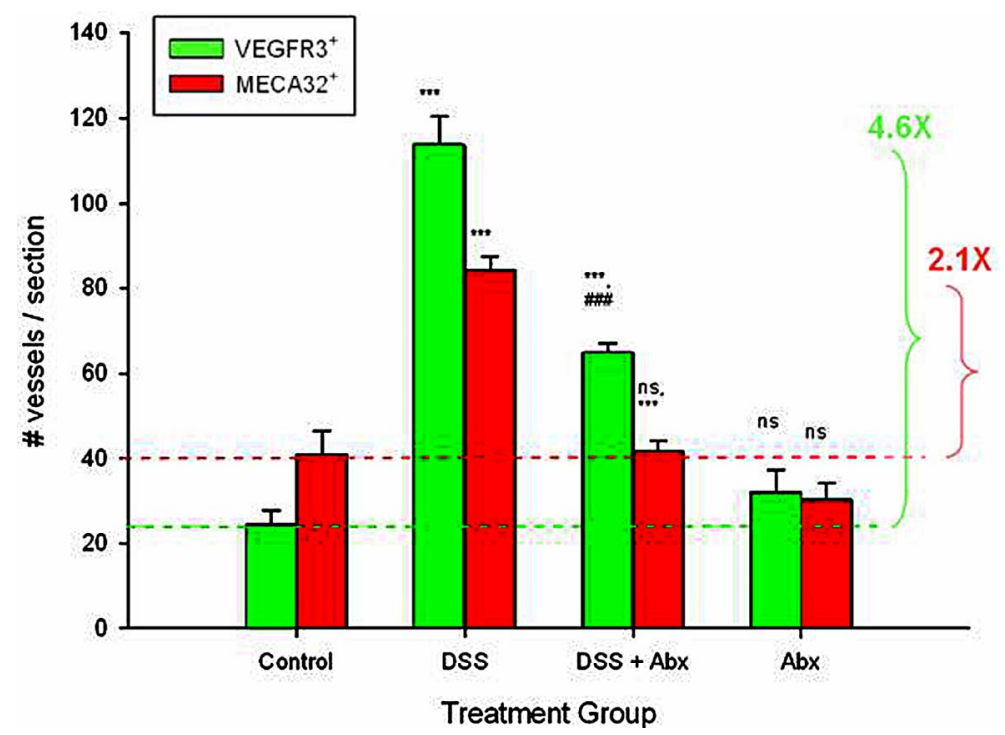

(c)

Figure 4. (a) MECA-32 stained blood vessels were significantly elevated in the colon of the DSS group (2.1-fold over control), while the ABX count was similar to the control group; (b) Lymphatic vessels (VEGFR-3) showed a 4.6-fold increase in DSS versus control, whereas the $\mathrm{ABX}$ group exhibited a 2.6 fold increase versus control; (c) Histogram of number of vessel/section (1-way ANOVA w/Bon-ferroni post testing).

\subsection{Effect of DSS on Trans-Epithelial Electrical Resistance (TEER)}

Depression in epithelial barrier function (Figure 6(a)) was found to be dependent on the dose of DSS. Controls showed only minor changes in barrier function, which increased slightly over 24 h. $0.5 \%$ DSS showed an initial drop in barrier, which recovered by $4 \mathrm{~h}$. At $4 \mathrm{~h}, 1 \%$ DSS showed a drop in barrier, but this also recovered by $24 \mathrm{~h}$. $3 \%$ DSS showed the largest drop in barrier, which progressively decreased to $84.1 \%$ of baseline by $24 \mathrm{~h}\left(^{* *} \mathrm{p}<\right.$ $0.01)$.

\subsection{Effect of DSS on Colonic Epithelial Cell Metabolism}

DSS also produced a dose-dependent and reversible de- crease in epithelial cell metabolism (Figure 6(b)). All DSS groups showed a significant decrease in metabolism at $4 \mathrm{~h}$, which was reversed during the recovery period, suggesting that DSS does not produce an irreversible injury to epithelial cells.

\section{DISCUSSION}

In recent years, animal experiments have demonstrated that gut commensal flora induced immunoinflammatory dysregulation, which contributes in the etiopathogenesis of colitis. While some forms of gut inflammation represent appropriate responses to abnormal or pathogenic gut flora, inflammation in IBD appears to result from an inappropriate response to normal gut flora. While many different antibiotics have been shown to decrease gut inflammation and colonic injury in human and animal 


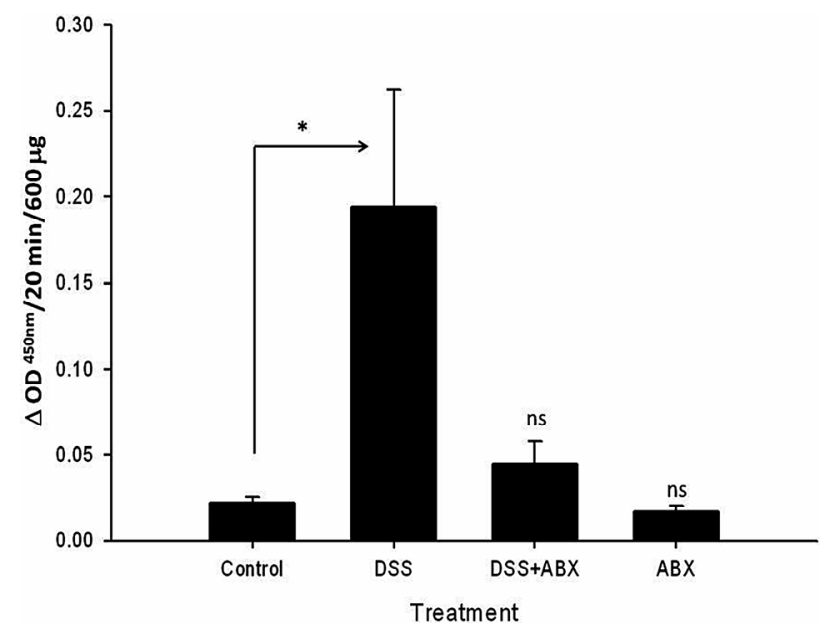

Figure 5. MPO content: Antibiotics suppress gut neutrophil infiltration in colitis. The level of necrophilia measured by MPO activity of the colonic tissue in the DSS group exhibited the highest myeloperoxidase activity versus that of the control group ( $<<0.05)$, Antibiotics reduced MPO activity in the DSS $+\mathrm{ABX}$ group compared to the DSS group.

models of colitis, the use of antibiotics in IBD treatment still remains controversial.

\subsection{Pro-Inflammatory Bacterial Trigger in Pathogenesis of IBD and Colitis}

It is interesting to note that normal commensal gut flora distribution in the intestine exhibits increased density towards the distal end of colon, containing both beneficial and potentially detrimental species. Lactobacillus and Bifidobacteria species have been found to exert antiinflammatory effects on the gut; administration of Lactobacillus-containing probiotics may even be beneficial in the treatment of UC and pouchitis [27-30]. Conversely, Bacteroides vulgates is a normal gut commensal which can initiate inflammation, while a high bacterial load of E. coli or Enterococcus can increase the intensity of gut inflammation [31]. Generally, many aerobes have been associated with local inflammation, while some anaerobes can create diffuse and severe fibrogenic transmural inflammation of the intestine [29]. Rakoff-Nahoum et al., however, have shown that commensal microflora are required for normal homeostasis and maintenance of intestinal epithelial integrity, yet it is unclear how the presence of normal microflora influences tissue injury during disturbances of normal intestinal epithelial barrier [32]. Our studies here show that gut sterilization (using Vancomycin, Metronidazole, and Neomycin) prevents the colonic injury and histopathological damages in the DSS + ABX group indicating that following injury, normal microflora can provoke localized injury responses, some of which may support resolution of this injury.

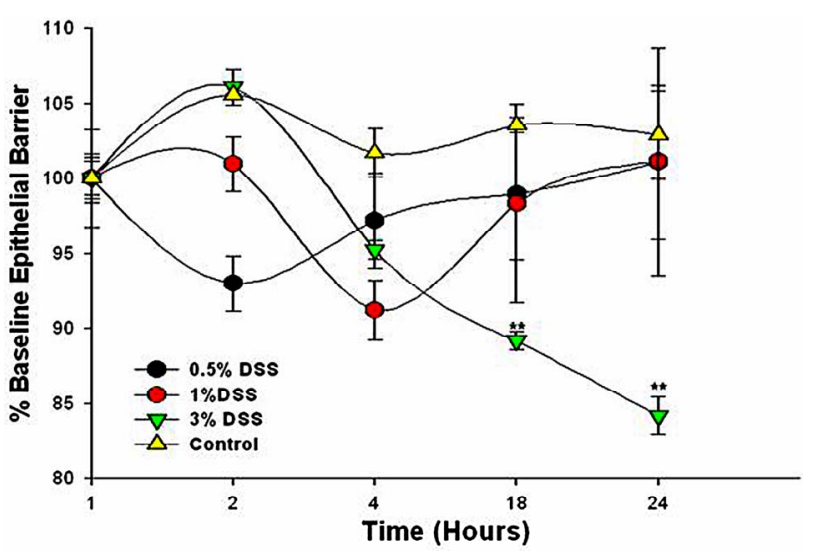

(a)

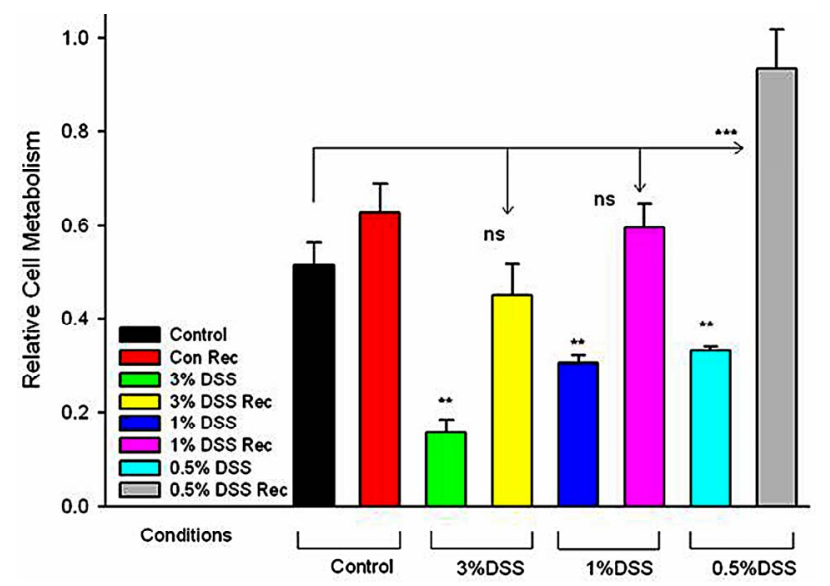

(b)

Figure 6. (a) DSS dysregulates epithelial barrier integrity: Epithelial electrical barrier changes in response to control, $0.5 \%$ DSS-treated, $1 \%$ DSS-treated, and 3\% DSS-treated groups are shown at 1, 2, 4, 18 and 24 hours after treatment; (b) DSS transiently dysregulates epithelial metabolism: Relative epithelial cell metabolism changes during different DSS concentration exposures are shown. Exposure phases display inhibition of cell metabolism in all DSS groups $\left(\mathrm{p}<0.01^{* *}\right)$ and during recovery period the $3 \%$ DSS and $1 \%$ DSS recovers similar to control.

\subsection{Antibiotic Treatment and Gut Barrier Dysfunction}

Changing the microbial mass or composition using antibiotics (particularly Metronidazole and Ciprofloxacin) has been effective for treating some Crohn's and UC patients, as well as IBD patients with septic complications [10,30]. Ciprofloxacin, Vancomycin-imipenem, and Amoxicillin/clavulanic acid have been shown to decrease inflammation in TNBS-induced colitis [33,34], while a combination of Metronidazole and Gentamicin is effective in reducing the number of translocated bacteria in acetic-acid induced colitis [35]. In mouse and rat studies, antibiotics have been successfully used to prevent the colitis that spontaneously develops in IL-10 deficient mice [13], to decrease the inflammation in DSS-induced 
colitis [36], to treat transgenic HLA-B27-related colitis [31], and to attenuate acetic acid colitis in mice [37]. These mouse and rat studies used combinations involving Ciprofloxacin, Metronidazole, Neomycin, Vancomycin, and Imipenem, but Rifaximin and Tobramycin have also had short-term success in UC [30]. When metronidazole and ciprofloxacin are used in combination, their efficacy is comparable to steroids (methylprednisolone) [30]. Neomycin and Metronidazole both established protection by an increase in level of Lactobacillus along with elimination of detrimental bacterial species [31], and vancomycin exhibits a role in prevention and reversed the established colitis in IL-10 mice.

Because gut barrier dysfunction can usually be shown to precede the development of gut inflammation [23], antibiotic clearance of non-beneficial commensals is particularly useful during instances of a diminished gut barrier in IBD. Although IL-10 gene deficient mice spontaneously develop colitis when colonized by normal gut commensals, these mice do not develop colitis when raised in a germ free environment; this suggests that foreign pathogens may also be involved in the process [38]. One well-known role of IL-10 is to regulate responses to the inflammatory Th1 cytokines IFN- $\gamma$ and TNF- $\alpha$, which can dysregulate the integrity of the gut epithelial cell layer. This leads to a compromised gut barrier in IL-10-/mice, and although etiology may be slightly different, similar pathology is also found in other animal models of IBD and in many cases of human IBD $[10,26]$. The barrier defect allows bacteria to gain access to the inner layers of the gut wall, as well as increase in numbers of adhesive bacteria, consequently leading to sustained inflammation. In fact, IL-10 deficient mice demonstrate a pre-existing increase in gut permeability to luminal contents even before colitis induction. Humans with IBD also exhibit decreased gut solute barrier function prior to development of inflammation [39]. In our study, even after DSS was used to disturb gut epithelial function, mice administered antibiotics failed to display significant changes in inflammation by a variety of measures; meanwhile, mice administered DSS without antibiotics did demonstrate clear signs of heavy inflammation. This finding furthers the notion that even when the epithelial barrier is disturbed, the progression of IBD requires the presence of bacteria.

Beneficial (e.g. pro-biotic) bacteria may also positively influence barrier through induction of Th2 cytokine responses and NF-kB inhibition. However, in the IL-10 KO model $[23,39]$, the inability to mobilize IL-10 may lead to a shift towards barrier failure as seen in human IBD [11]. We found that DSS produced a rapid and reversible depression in gut epithelial metabolism, which was correlated with barrier changes. Therefore, DSS ap- pears to produce a transient barrier disturbance that allows the penetration of bacterial antigens into the lamina propria to provoke inflammation. However, because epithelia appear to be relatively intact in colons of DSS + ABX treated mice on day 11, (Figure 2(a)) our findings suggest that commensal antigens trigger gut inflammation that leads to epithelial injury mediated by infiltrated leukocytes, rather than simple chemical erosion of the epithelia. This is supported by histologic maintenance of colonic epithelia in DSS + ABX treated mice.

\subsection{Histopathological Analysis, MPO Content, and Attenuation of Inflammation}

The most direct measure of intestinal inflammation and damage is gross and microscopic examination of the colons and spleens. Control and ABX colons showed the normal colonic epithelial architecture with no evidence of inflammation and edema of intestine. However, DSS mice had significantly shorter, heavier, and larger colons, as well as heavier spleens. The addition of antibiotics substantially lowered these measures of inflammation, as the sizes of the colons and spleens in DSS + ABX mice, which suggests a reduction of disease activity. The microscopic examination resulted in similar findings; antibiotic administration was associated with decreased inflammation and histological damage.

Light microscopy demonstrated prominent involvement of the distal colon with respect to the mid- and proximal colon, which becomes more extensively involved in the DSS group. Presence of atrophy, crypt abscesses, and distortion indicate severe inflammation in the distal colon. Human UC is also characterized by inflammatory changes mainly limited to the rectum and to the left colon and confined within the mucosa. The lamina propria appears edematous, with vascular congestion and the presence of mixed inflammatory infiltrates (granulocytes, lymphocytes, and plasma cells) as well as cryptitis and crypt abscesses with depletion of goblet cells, crypt architecture distortion, and lymphoid aggregates. Severe human IBD also shows inflammatory changes, with shortening of the colon as a result of muscular contraction. Together, these factors confirm the similarities between human ulcerative colitis and mouse DSS colitis.

Neutrophil infiltration is an index of acute inflammation. DSS-induced chemical inflammation triggers the infiltration of neutrophils and subsequently chemokines, cytokines, growth factors and proteolytic enzymes, which contributes to colonic injury and new blood and lymphatic vessel formation. Decrement effect of antibiotics in MPO content prevents significant colonic inflammation in the DSS + ABX group, explaining the preserved histologic integrity of the colon. 


\subsection{Modulation of Angiogenesis and Lymphangiogenesis with Gut Sterilization}

We found that DSS-induced inflammation leads to increased gut angiogenesis and lymphangiogenesis, likely as a result of elevated levels of angiogenic factors like VEGF-C and VEGF-A in colitis [40,41]. The rise in vessel density in the DSS-treated colon was correlated with notable pathological changes in the colon, while the antibiotic-treated DSS group demonstrated a complete blockade of inflammatory angiogenesis, as well as a substantial decrease in lymphangiogenesis, possibly by preventing the remodeling of vascular tissue by ang1/ang2 regulation. The ratio of lymphatic to blood vessel density was twice as high in the DSS group $(\mathrm{L} / \mathrm{B}=1.3)$ than in the control group $(\mathrm{L} / \mathrm{B}=0.61)$, although this ratio was increased further $(\mathrm{L} / \mathrm{B}=1.55)$ in the DSS $+\mathrm{ABX}$ treated group. This is likely a consequence of the fact that blood vessel expansion mediates injury, while lymphatic vessel injury does not; conversely, increased lymphatic density appears to adaptively balance interstitial fluid accumulation, especially when epithelial barrier is altered (e.g. by DSS, Figure 6(a)).

Interestingly, at least part of the benefit of UC therapy using mesalamine (5-ASA) may be due to the normalizetion of the balance between angiogenic/ anti-angiogenic factors. In iodoacetamide induced ulcerative colitis, 5ASA increased angiostatin/endostatin levels and decreased TNF- $\alpha$, which down-regulates MMP-2 and MMP-9 [42]. Angiopoietin-2 is an important ligand of the Tie-2 receptor, which dysregulates blood and lymphatic vascular networks in both humans and mice. We reported in our previous study [43] that Ang-2-/- mice treated with DSS exhibited no remodeling of blood or lymphatic vessels, with significantly fewer infiltrated leukocytes but still exhibited increased gut fragility (occult blood), disease activity, weight loss, and diarrhea. While angiogenesis knockout may limit events in gut inflammation, simultaneous interference with lymphatics mediated by Ang-2-/appears to eliminate some or all of the benefit of antihemangiogenesis (blood vessel remodeling). Several studies on angiogenesis blockade in IBD shows a decrease in DAI, histopathology and angiogenesis in IL-10-/- mice when treated with the hemangiogenesis blocker ATN-161 [44]. It is important to note that in the current study we find that antibiotic protection against injury reflects complete block in blood vessel growth, but persistence of lymphatic vascular growth. This appears to be most consistent with new blood vessel growth rather than lymphatic growth as mediating injury in experimental colitis.

The present study reveals certain characteristic effects of antibiotics (by removing commensals) on the complex acute inflammatory response on new blood and lymphatic vessel formation. The findings suggest that elimi- nation of gut flora by antibiotics reduces injury and prevents leukocyte infiltration into the gut. Injury reduction is correlated with a complete blockade of neo-angiogenesis. These findings were further correlated with a reduction, but not elimination, of lymphangiogenesis. These findings support lymphatic expansion in colitis as a protective feature of gut injury which interacts with angiogenesis. Therapies to enhance lymphangiogenesis or normalize lymphatic function may improve clinical responses.

Antibiotics have been shown to ameliorate some features of IBD, and reduce the amount of inflammatory damage to the mouse intestine; however, they have not previously been directly related to inflammatory angiogenesis, an important new mechanism in IBD therapy. Further our data supports the protective ability of lymphangiogenesis against development of injury in this model. Therefore, in the future, antibiotics may be combined with anti-angiogenesis agents, as well as prolymphangiogenic agents, to prevent development or exacerbation of human IBD.

\section{CONCLUSION}

Diverse IBDs share immune dysregulation toward normal gut bacteria and altered gut barrier function. A reduction in gut bacteria, despite barrier disturbances, might lessen deleterious effects of the disease. Broad spectrum antibiotics decreased colon neutrophil infiltration, disease symptom severity and histopathological evidence of colitis. Antibiotics completely prevented inflammatory angiogenesis and partially reduced inflammation associated lymphangiogenesis, which may be important events both in the development and regulation of inflammation. Our data suggests that infiltration of bacterial antigens into the gut lamina propria is necessary for the initiation of angiogenesis. Lymphangiogenesis was induced despite prevention of colon injury and suggests that unlike angiogenesis, lymphangiogenesis may represent an adaptive response in colon inflammation.

\section{ACKNOWLEDGEMENTS}

We acknowledge Shannon Wells for support and assistance in laboratory, histology and experimental studies. We also acknowledge support from LSU Health Shreveport Departments of Molecular \& Cellular Physiology, Cellular Biology \& Anatomy, Gastroenterology \& Hepatology, and Microbiology \& Immunology. We also acknowledge Courtney Parker, Samir Patel and Shan Siddiqui for their assistance in editing and formatting the manuscript.

\section{REFERENCES}

[1] Jantchou, P., Monnet, E. and Carbonnel, F. (2006) Environmetal risk factors in Crohn's disease and ulcerative colitis (excluding tobacco and appendicectomy). Gastro- 
entérologie Clinique et Biologique, 30, 859-867. doi:10.1016/S0399-8320(06)73333-4

[2] Veluswamy, H., Suryawala, K., Sheth, A., Wells, S., Salvatierra, E., Cromer, W., Chaitanya, G.V., Painter, A., Patel, M., Manas, K., Zwank, E., Boktor, M., Baig, K., Datti, B., Mathis, M.J., Minagar, A., Jordan, P.A. and Alexander, J.S. (2010) African-American inflammatory bowel disease in a Southern US health center. BMC Gastroenterology, 10, 104. doi:10.1186/1471-230X-10-104

[3] Buhner, S., Buning, C., Genschel, J., Kling, K., Herrmann, D., Dignass, A., Kuechler, I., Krueger, S., Schmidt, H.H. and Lochs, H. (2006) Genetic basis for increased intestinal permeability in families with Crohn's disease: Role of CARD15 3020insC mutation? Gut, 55, 342-347. doi:10.1136/gut.2005.065557

[4] Sartor, R.B. and Muehlbauer, M. (2007) Microbial host interact-tions in IBD: Implications for pathogenesis and therapy. Current Gastroenterology Reports, 9, 497-507. doi:10.1007/s11894-007-0066-4

[5] Reuter, B.K. and Pizarro, T.T. (2009) Mechanisms of tight junction dysregulation in the SAMP1/YitFc model of Crohn's disease-like ileitis. Annals of the New York Academy of Sciences, 1165, 301-307. doi:10.1111/j.1749-6632.2009.04035.x

[6] Cromer, W., Jennings, M.H., Odaka, Y., Mathis, J.M. and Alexander, J.S. (2010) Murine rVEGF164b, an inhibitory VEGF reduces VEGF-A-dependent endothelial proliferation and barrier dysfunction. Microcirculation, 17, 536547.

[7] Podolsky, D.K. (1997) Lessons from genetic models of inflammatory bowel disease. Acta Gastroenterologica Belgica, 60, 163-165.

[8] Laukoetter, M.G., Nava, P. and Nusrat, A. (2008) Role of the intestinal barrier in inflammatory bowel disease. World Journal of Gastroenterology, 14, 401-407. doi:10.3748/wjg.14.401

[9] Soriano, A., Salas, A., Salas, A., Sans, M., Gironella, M., Elena, M., Anderson, D.C., Pique, J.M. and Panes, J. (2000) VCAM-1, but not ICAM-1 or MAdCAM-1, immunoblockade ameli rates DSS-induced colitis in mice. Laboratory Investigation, 80, 1541-1551. doi:10.1038/labinvest.3780164

[10] Oshima, T., Laroux, F.S., Coe, L.L., Morise, Z., Kawachi, S., Bauer, P., Grisham, M.B., Specian, R.D., Carter, P., Jennings, S., Granger, D.N., Joh, T. and Alexander, J.S. (2001) Interferon gamma and interleukin-10 reciprocally regulate endothelial junction integrity and barrier function. Microvascular Research, 61, 130-143. doi:10.1006/mvre.2001.2322

[11] Sydora, B.C., Martin, S.M., Lupicki, M., Dieleman, L.A., Doyle, J., Walker, J.W. and Fedorak, R.N. (2006) Bacterial antigens alone can influence intestinal barrier integrity, but live bacteria are required for initiation of intestinal inflammation and injury. Inflammatory Bowel Disease, 12, 429-436. doi:10.1097/00054725-200606000-00001

[12] Tellez, H.S. (2010) Digestive physiology and the role of microorganisms. Journal of Applied Poultry Research, 15, 136-144.
[13] Madsen, K.L., Doyle, J.S., Tavernini, M.M., Jewell, L.D., Rennie, R.P. and Fedorak, R.N. (2000) Antibiotic therapy attenuates coli-tis in interleukin 10 gene-deficient mice. Gastroenterology, 118, 1094-1105. doi:10.1016/S0016-5085(00)70362-3

[14] Wold, J.S. and Turnipseed, S.A. (1981) Toxicology of vancom-ycin in laboratory animals. Reviews of Infection Disease, 3, S224-S229. doi:10.1093/clinids/3.Supplement 2.S224

[15] Dieleman, L.A., Pena, A.S., Meuwissen, S.G. and Van Rees, E.P. (1997) Role of animal models for the pathogenesis and treatment of inflammatory bowel disease. Scandanavian Journal of Gastroenterology, 223, 99-104.

[16] Kim, H.S. and Berstad, A. (1992) Experimental colitis in animal models. Scandanavian Journal of Gastroenterology, 27, 529-537. doi:10.3109/00365529209000116

[17] Sasaki, M., Bharwani, S., Jordan, P., Elrod, J.W., Grisham, M.B., Jackson, T.H., Lefer, D.J. and Alexander, J.S. (2003) Increased disease activity in eNOS-deficient mice in experimental colitis. Free Radical Biology and Medicine, 35, 1679-1687. doi:10.1016/j.freeradbiomed.2003.09.016

[18] Sasaki, M., Bharwani, S., Jordan, P., Joh, T., Manas, K., Warren, A., Harada, H., Carter, P., Elrod, J.W., Wolcott, M., Grisham, M.B. and Alexander, J.S. (2003) The 3-hydroxy-3-methylglutaryl-CoA reductase inhibitor pravastatin reduces disease activity and inflammation in dextran-sulfate induced colitis. Journal of Pharmacology and Experimental Therapeutics, 305, 78-85. doi:10.1124/jpet.102.044099

[19] Sasaki, M., Mathis, J.M., Jennings, M.H., Jordan, P., Wang, Y., Ando, T., Joh, T. and Alexander, J.S. (2005) Reversal of experimental colitis disease activity in mice following administration of an adenoviral IL-10 vector. Journal of Inflammation, $2,13$. doi:10.1186/1476-9255-2-13

[20] Cooper, H.S., Murthy, S.N., Shah, R.S. and Sedergran, D.J. (1993) Clinicopathologic study of dextran sulfate sodium exper mental murine colitis. Laboratory Investigation, 69, 238-249.

[21] Grisham, M.B., Granger, D.N. and Lefer, D.J. (1998) Modulation of leukocyte-endothelial interactions by reactive metabolites of oxygen and nitrogen: Relevance to ischemic heart disease. Free Radical Biology and Medicine, 25, 404-433. doi:10.1016/S0891-5849(98)00094-X

[22] Hausmann, M., Obermeier, F., Paper, D.H., Balan, K., Dunger, N., Menzel, K., Falk, W., Schoelmerich, J., Herfarth, H. and Rogler, G. (2007) In vivo treatment with the herbal phenyletanoid acteoside ameliorates intestinal inflammation in dextran sulphate sodium-induced colitis. Clinical \& Experimental Immunology, 148, 373-381. doi:10.1111/j.1365-2249.2007.03350.x

[23] Arrieta, M.C., Madsen, K., Doyle, J. and Meddings, J. (2009) Reducing small intestinal permeability attenuates colitis in the IL10 gene-deficient mouse. Gut, 58, 41-48. doi:10.1136/gut.2008.150888

[24] Lee, Y.T., Sung, J.J., Poon, P., Lai, K.N. and Li, P.K. (1998) Association of HLA class-II genes and anti-neutrophil cytoplasmic antibodies in Chinese patients with 
inflammatory bowel disease. Scandanavian Journal of Gastroenterology, 33, 623-627. doi:10.1080/00365529850171909

[25] Mandriota, S.J., Jussila, L., Jeltsch, M., Compagni, A., Baetens, D., Prevo, R., Banerji, S., Huarte, J., Montesano, R., Jackson, D.G., Orci, L., Alitalo, K., Christofori, G. and Pepper, M.S. (2001) Vascular endothelial growth factor-C-mediated lymphan-giogenesis promotes tumour metastasis. $E M B O, \mathbf{2 0}, 672-682$. doi:10.1093/emboj/20.4.672

[26] McGuckin, M.A., Eri, R., Simms, L.A., Florin, T.H. and Radford-Smith, G. (2009) Intestinal barrier dysfunction in inflammatory bowel diseases. Inflammatory Bowel Disease, 15, 100-113. doi:10.1002/ibd.20539

[27] Cummings, J.H., Macfarlane, G.T. and Macfarlane, S. (2003) Intestinal bacteria and ulcerative colitis. Current Issues in Intestinal Microbiology, 4, 9-20.

[28] Dieleman, L.A., Goerres, M.S., Arends, A., Sprengers, D., Torrice, C., Hoentjen, F., Grenther, W.B. and Sartor, R.B. (2003) Lactob cillus GG prevents recurrence of colitis in HLA-B27 transgenic rats after antibiotic treatment. Gut, 52, 370-376. doi:10.1136/gut.52.3.370

[29] Guarner, F. and Malagelada, J.R. (2003) Role of bacteria in ex perimental colitis. Best Practice \& Research Clinical Gatroenterology, 17, 793-804. doi:10.1016/S1521-6918(03)00068-4

[30] Kanauchi, O., Mitsuyama, K., Araki, Y. and Andoh, A. (2003) Modification of intestinal flora in the treatment of inflammatory bowel disease. Current Pharmaceutical Design, 9, 333-346. doi:10.2174/1381612033391883

[31] Rath, H.C., Schultz, M., Freitag, R., Dieleman, L.A., Li, F., Linde, H.J., Scholmerich, J. and Sartor, R.B. (2001) Different subsets of enteric bacteria induce and perpetuate experimental colitis in rats and mice. Infection and Immunity, 69, 2277-2285. doi:10.1128/IAI.69.4.2277-2285.2001

[32] Rakoff-Nahoum, S., Paglino, J., Eslami-Varzaneh, F., Edberg, S. and Medzhitov, R. (2004) Recognition of commensal micro-flora by toll-like receptors is required for intestinal home-ostasis. Cell, 118, 229-241. doi:10.1016/j.cell.2004.07.002

[33] Lahat, G., Halperin, D., Barazovsky, E., Shalit, I., Rabau, M., Klausner, J. and Fabian, I. (2007) Immunomodulatory effects of ciprofloxacin in TNBS-induced colitis in mice. Inflammatory Bowel Disease, 13, 557-565.

[34] Videla, S., Vilaseca, J., Guarner, F., Salas, A., Treserra, F., Crespo, E., Antolin, M. and Malagelada, J.R. (1994) Role of Intestinal microflora in chronic inflammation and ulceration of the rat colon. Gut, 35, 1090-1097. doi:10.1136/gut.35.8.1090

[35] Yigitler, C., Gulec, B., Aydogan, H., Ozcan, A., Kilinc, M., Yigit, T., Kozak, O. and Pekcan, M. (2004) Effect of mesalazine, metronidazole and gentamicin on bacterial translocation in experimental colitis. Journal of Gastroenterology and Hepatology, 19, 1179-1186. doi:10.1111/j.1440-1746.2004.03457.x

[36] Hans, W., Scholmerich, J., Gross, V. and Falk, W. (2000) The role of the resident intestinal flora in acute and chronic dextran sulfate sodium-induced colitis in mice. European Journal of Gastroenterology \& Hepatology, 12, 267-273. doi:10.1097/00042737-200012030-00002

[37] Kang, S.S., Bloom, S.M., Norian, L.A., Geske, M.J., Flavell, R.A., Stappenbeck, T.S. and Allen, P.M. (2008) An antibiotic responsive mouse model of fulminant ulcerative coltis. Public Library of Science Medicine, 5, e41.

[38] Hoentjen, F., Harmsen, H.J., Braat, H., Torrice, C.D., Mann, B.A., Sartor, R.B. and Dieleman, L.A. (2003) Antibiotics with a seletive aerobic or anaerobic spectrum have different therapeutic activities in various regions of the colon in interleukin 10 gene deficient mice. Gut, 52, 1721-1727. doi:10.1136/gut.52.12.1721

[39] Werner, T. and Haller, D. (2007) Intestinal epithelial cell signaling and chronic inflammation: From the proteome to specific molecular mechanisms. Mutation Research, 622, 42-57. doi:10.1016/j.mrfmmm.2007.05.010

[40] Binion, D.G., Otterson, M.F. and Rafiee, P. (2008) Curcumin inhi its VEGF-mediated angiogenesis in human intestinal mcrovascular endothelial cells through COX-2 and MAPK inhibition. Gut, 57, 1509-1517. doi:10.1136/gut.2008.152496

[41] Goebel, S., Huang, M., Davis, W.C., Jennings, M., Siahaan, T.J., Alexander, J.S. and Kevil, C.G. (2006) VEGFA stimulation of leukocyte adhesion to colonic microvascular endothelium: Implications for inflammatory bowel disease. American Journal of Physiology-Gastrointestinal and Liver Physology, 290, G648-G654.

[42] Deng, X., Tolstanova, G., Khomenko, T., Chen, L., Tarnawski, A., Szabo, S. and Sandor, Z. (2009) Mesalamine restores angiogenic balance in experimental ulcerative colitis by reducing expression of endostatin and angiostatin: Novel molecular mechanism for therapeutic action of mesalmine. Journal of Pharmacology and Experimental Therapeutics, 331, 1071-1078. doi:10.1124/jpet.109.158022

[43] Ganta, V.C., Cromer, W., Mills, G.L., Traylor, J., Jennings, M., Daley, S., Clark, B., Mathis, J.M., Bernas, M., Boktor, M., Jordan, P., Witte, M. and Alexander, J.S. (2010) Angiopoietin-2 in experimental colitis. Inflammatory Bowel Disease, 16, 1029-1039. doi:10.1002/ibd.21150

[44] Danese, S., Sans, M., Spencer, D.M., Beck, I., Donate, F., Plunkett, M.L., de la, M.C., Redline, R., Shaw, D.E., Levine, A.D., Mazar, A.P. and Fiocchi, C. (2007) Angiogenesis blockade as a new therapeutic approach to experimental colitis. Gut, 56, 855-862. doi:10.1136/gut.2006.114314 